\title{
Response to: "Sarcopenia and Back Muscle Degeneration as Risk Factors for Back Pain: A Comparative Study"
}

\author{
Whoan Jeang Kim, Kap Jung Kim, Dae Geon Song, Jong Shin Lee, \\ Kun Young Park, Jae Won Lee, Shann Haw Chang, Won Sik Choy \\ Department of Orthopedic Surgery, Eulji University Hospital, Daejeon, Korea
}

Dear Sir,

We appreciate the letter regarding our manuscript titled "Sarcopenia and back muscle degeneration as risk factors for back pain: a comparative study [1]." We thank the readers who are interested in our article and provided valuable feedback. Our reply to comments is as follows:

Question: The Asian Spine Journal recently published an article in volume 14(3), 2020 by Kim et al. [1] "Sarcopenia and back muscle degeneration as risk factors for back pain: a comparative study." The authors have read the article with great interest. The introduction is well written. Also, the author had well explained about degree of back pain association with back muscle degeneration than with sarcopenia. They developed back muscle degeneration risk index, indicating it to be a useful parameter for evaluation of back pain and muscle degeneration. We gathered valuable information and congratulate the authors for this. However, there are some issues, which needs clarification.

The sample size of the study was not determined which is needed for deriving meaning conclusion. If the sample size is less than the optimum sample size, even the most thoroughly executed study may estimate those effects or associations too imprecisely. And, if the sample size is too large, may even lead to a loss in accuracy [2]. Also, also the subjects were taken from one center so, identified risk factors may be unique to that single center. This methodological choice may weaken the generalizability of the study findings [3].

Our reply: The effect size for each group was set to 0.8 [4]. Assuming an acceptable alpha error of 0.5 , aiming for the 95\% power of the study and two-tailed hypothesis was used $[5,6]$. And allocation ratio of each group comparison in our paper was $1,1.12,1.79,2.73$, and 4.58 , respectively. The calculated effect total sample size was found to be 84 , $84,94,106$, and 140, respectively. Participants presented in our study are all exceed effect total sample size.

Question: The author did not mention about normality test. Though, for meaningful conclusions, assumption of the normality should be followed regardless of the sample size. Choosing the wrong representative data and calculating significance level using the value of data set might give erroneous interpretation. If appropriate, then parametric test is used to compare means, otherwise medians are

Received Jun 25, 2020; Accepted Jun 25, 2020

Corresponding author: Dae Geon Song

Department of Orthopedic Surgery, Eulji University Hospital, 95 Dunsanseo-ro, Seo-gu, Daejeon 35233, Korea

Tel: +82-42-611-3267, Fax: +82-42-259-1289, E-mail: jaystar4u@naver.com 
used to compare the groups, using nonparametric methods [7]. SPSS provides the Kolmogorov-Smirnov test for normality test of sample size $>50$ [8].

The authors have reported that comparison of backpain severity between sarcopenia/non-sarcopenia and high/low back muscle degeneration groups was compared by Student $t$-test or Mann-Whitney $U$-test. The statement is confusing regarding use of parametric or non parametric test at the same time.

Our reply: Thank you for your comment. First, all participants of each group in our study were exceed 30 , so we didn't mention about normality test in the literature. Kolmogorov-Smirnov test was used as a test of normality via IBM SPSS ver. 22.0 software (IBM Corp., Armonk, NY, USA) [8]. In all groups, the $p$-value was calculated more than 0.05 and the null hypothesis that it follows the normal distribution was not rejected. So, we used parametric two-sample independent $t$-test (unpaired Student $t$-test) in comparison of all groups. We apologize for the confusion by mentioning Mann-Whitney $U$-test in the literature.

Question: Results reported that there was greater prevalence of sarcopenia in back pain group. Type 2 diabetes mellitus is associated with sarcopenia and frailty [9]. Studies have found a link between hyperglycemia and the biochemical events that may provide a potential mechanism by which diabetes may contribute to back pain [10]. There has been no mention of history taking or interview or measurement of diabetes in the subjects. There may be a probability that back pain patient group may have more number of diabetes subjects compared to controls contributing to muscle weakness leading to pain and sarcopenia.

Our reply: Your comment is acceptable and reasonable. As we mentioned in study limitations, the etiology of back pain is so variable that it is difficult to know exactly whether a given condition such as myofasciitis, diabetes mellitus, disc degeneration, facet joint degeneration, etc. This heterogeneity might weaken our findings. But we analyzed the effect of sarcopenia and back muscle degeneration on back pain at the same time. Future studies need to classify patients by etiology of back pain and this will clarify the findings.

Question: Next under discussion authors report regard- ing reliability testing of lumbar extensor muscles on 30 subjects though there was no aim mentioned like this nor anything of such kind was mentioned under methodology. Neither under subject recruitment and methodology, authors mention about 30 more participants for reliability testing and procedure for reliability testing of lumbar extensor muscles.

The study provides valuable information but the abovementioned points need to be considered for clinical interpretation.

Our reply: We mentioned methodology on measuring lumbar extensor strength in Materials and Methods section (Lumbar extensor strength was measured using a lumbar extension dynamometer in the sitting position with stable pelvis. Warm-up trials and stretching exercises were conducted for 5 minutes before measurements. The testing device was set to an angular velocity of $60^{\circ}$ per second, and lumbar extensor strength was measured 5 times and recorded as peak torque $[\mathrm{Nm}]$ ). Data for measuring intra-class correlation coefficient were obtained using 30 separate participants and calculated using IBM SPSS ver. 22.0 software (IBM Corp.). We obtained excellent reliability (intraclass correlation coefficient $=0.862$ ) according to Cicchetti [11]. Moreover, the isokinetic lumbar extensor dynamometer is widely used and its reliability well established $[12,13]$.

\section{Conflict of Interest}

No potential conflict of interest relevant to this article was reported.

\section{References}

1. Kim WJ, Kim KJ, Song DG, et al. Sarcopenia and back muscle degeneration as risk factors for back pain: a comparative study. Asian Spine J 2020;14:36472.

2. Zodpey SP. Sample size and power analysis in medical research. Indian J Dermatol Venereol Leprol 2004;70:123-8.

3. Song JW, Chung KC. Observational studies: cohort and case-control studies. Plast Reconstr Surg 2010;126:2234-42.

4. Cohen J. A power primer. Psychol Bull 1992;112:1559. 
5. Faul F, Erdfelder E, Lang AG, Buchner A. G*Power 3: a flexible statistical power analysis program for the social, behavioral, and biomedical sciences. Behav Res Methods 2007;39:175-91.

6. Faul F, Erdfelder E, Buchner A, Lang AG. Statistical power analyses using $G^{\star}$ Power 3.1: tests for correlation and regression analyses. Behav Res Methods 2009;41:1149-60.

7. Mishra P, Pandey CM, Singh U, Gupta A, Sahu C, Keshri A. Descriptive statistics and normality tests for statistical data. Ann Card Anaesth 2019;22:67-72.

8. Ghasemi A, Zahediasl S. Normality tests for statistical analysis: a guide for non-statisticians. Int J Endocrinol Metab 2012;10:486-9.

9. Umegaki H. Sarcopenia and diabetes: hyperglycemia is a risk factor for age-associated muscle mass and functional reduction. J Diabetes Investig 2015;6:6234.
10. Rinaldo L, McCutcheon BA, Gilder H, et al. Diabetes and back pain: markers of diabetes disease progression are associated with chronic back pain. Clin Diabetes 2017;35:126-31.

11. Cicchetti DV. Guidelines, criteria, and rules of thumb for evaluating normed and standardized assessment instruments in psychology. Psychol Assess 1994;6:284-90.

12. Drouin JM, Valovich-mcLeod TC, Shultz SJ, Gansneder BM, Perrin DH. Reliability and validity of the Biodex system 3 pro isokinetic dynamometer velocity, torque and position measurements. Eur J Appl Physiol 2004;91:22-9.

13. Nordez A, Casari P, Cornu C. Accuracy of Biodex system 3 pro computerized dynamometer in passive mode. Med Eng Phys 2008;30:880-7. 